\title{
Adequação do teste de condutividade elétrica para sementes de Pisum sativum subsp. Arvense
}

\author{
Suitability of electrical conductivity test for seeds of Pisum sativum subsp. Arvense
}

\author{
Carla Gomes Machado ${ }^{I}$ Cibele Chalita MartinsII Denise Garcia de Santana ${ }^{\text {III }}$ \\ Simério Carlos Silva Cruz ${ }^{I}$ Silvia Sanielle Costa de Oliveira ${ }^{\text {I }}$
}

\section{RESUMO}

A qualidade da semente na produção agrícola é um dos principais fatores a ser considerado na implantação da cultura, de forma que se torna importante a obtenção de informações sobre a germinação e o vigor das sementes, além da necessidade de avaliá-los. Dentro desse contexto, este trabalho teve como objetivo adequar a metodologia do teste de condutividade elétrica para a avaliação da qualidade fisiológica de sementes de Pisum sativum subsp. arvense. Para tanto, foram utilizados dez lotes de sementes da cultivar IAPAR 83, empregando-se períodos de condicionamento de 8, 16, 20, 24 e 28 horas, combinados às temperaturas de 20 e $25^{\circ} \mathrm{C}$ e volumes de 75 e $250 \mathrm{~mL}$ de água. Além destes, foram conduzidos os testes de germinação, primeira contagem de germinação e emergência de plântulas. Para ambas as avaliações, foram utilizadas quatro repetições de 50 sementes. Os testes de vigor, assim como o teste de germinação foram sensíveis para avaliar a qualidade das sementes dos diferentes lotes estudados, porém houve variações na ordenação deles quanto ao vigor. $O$ volume de água, o tempo e a temperatura de embebição influenciaram os valores de condutividade elétrica. Concluiu-se que o teste de condutividade elétrica utilizando $250 \mathrm{~mL}$ de água, na temperatura de $25^{\circ} \mathrm{C}$ por 24 horas é promissor para a diferenciação de lotes de sementes de P. sativum subsp. arvense.

Palavras-chave: ervilha forrageira, vigor, potencial fisiológico, lixiviação.

\section{ABSTRACT}

Seed quality in agricultural production is a major factor to be considered in the deployment of a crop, so it

\begin{abstract}
becomes important to obtain information about seed vigor and germination and. This study had the objective to adjust the methodology of the electrical conductivity test to evaluate the physiological quality of Pisum sativum subsp. arvense seeds. Ten lots of the cultivar 'IAPAR 83' were studied to establish the methodology for the electrical conductivity test. It was studied germination, first count of germination and seedling emergence in greenhouse. For the electrical conductivity test, different temperatures $\left(20^{\circ} \mathrm{C}\right.$ and $\left.25^{\circ} \mathrm{C}\right)$, water volumes $(75 \mathrm{~mL}$ and 250mL) and imbibition periods (8, 16, 20, 24 and 28 hours) were evaluated. For both evaluations, four replications of 50 seeds were used. The vigor and germination tests were sensitive to evaluate the quality of seeds from different studied lots, although there were variations in the ordering of the lots with respect to their vigor. The water volume, time and soaking temperature influenced the electrical conductivity values. It was concluded that the electrical conductivity test is sensitive to differentiate seed lots of forage pea whenever conducted with $250 \mathrm{~mL}$ of water under $25^{\circ} \mathrm{C}$ for 24 hours.
\end{abstract}

Key words: forage pea, vigor, physiological potential, leaching.

\section{INTRODUÇÃO}

A produção de ervilhas no Brasil tem como principal objetivo a alimentação humana, porém, com o estudo das ervilhas de tegumento marrom (Pisum sativum subsp. arvense), verificou-se o potencial e a adaptação destes materiais visando o arraçoamento animal. Esse genótipo, chamado de ervilha forrageira

'Unidade Universitária de Palmeiras de Goiás, Universidade Estadual de Goiás (UEG), 76190-000, Palmeiras de Goiás, GO, Brasil, E-mail: carlagomesmachado@gmail.com. Autor para correspondência.

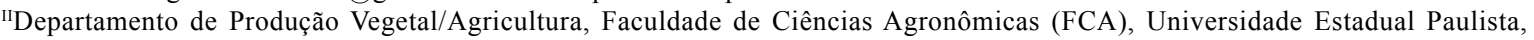
(UNESP), Botucatu, SP, Brasil.

IIIInstituto de Ciências Agrárias, Universidade Federal de Uberlândia (UFU), Uberlândia, MG, Brasil. 
constitui cultura anual para uso como forragem, adubação verde e cobertura do solo, com características importantes para a conservação e fertilidade do solo, sendo cultivada no inverno, quando muitas áreas agrícolas permanecem sem uso no Brasil. A aptidão dos grãos para a formulação de ração animal pode ser uma alternativa devido os altos níveis de proteína bruta (GIORDANO \& PEREIRA, 1989).

Para sementes comerciais de $\boldsymbol{P}$. sativum subsp. arvense, regulamentadas pelos padrões de sementes, as exigências são menores quanto à qualidade dos lotes em relação às grandes culturas e olerícolas, o que deve-se ao fato de a tecnologia de produção de sementes dessa espécie ser menos desenvolvida em relação às outras espécies cultivadas há mais tempo.

$\mathrm{Na}$ avaliação da qualidade fisiológica das sementes, rotineiramente é usado o teste de germinação (BRASIL, 2009) e, de modo complementar, são utilizados os resultados das análises de vigor para identificar diferenças na qualidade fisiológica dos lotes, principalmente daqueles com porcentagem semelhante de plântulas normais (VIEIRA \& CARVALHO, 1994). Esse tipo de informação pode ajudar na tomada de decisões internas das empresas produtoras de sementes quanto ao destino de determinado lote (MARCOS FILHO, 2005).

Dentre os testes de vigor atualmente utilizados, o teste de condutividade elétrica tem se destacado por sua facilidade de execução, baixo custo, rapidez, repetibilidade e fácil interpretação de resultados, sendo que se baseia no princípio de que as membranas celulares das sementes são as últimas a se organizarem durante o processo de maturação, no entanto, são as primeiras com sinais de deterioração após as sementes alcançarem o estádio de maturidade fisiológica (MARCOS FILHO, 2005). Quando sementes secas entram em contato com a água durante a embebição, ocorre rápida e intensa lixiviação de eletrólitos, proporcional ao estado de desorganização das membranas, seguida de redução na perda de solutos, à medida que os tecidos são reidratados, até atingir o equilíbrio (ROSA et al., 2000).

Assim, sementes mais deterioradas ou danificadas liberam maiores quantidades de lixiviados durante a embebição, refletindo o grau de desorganização de suas membranas e, consequentemente, caracterizando sua qualidade fisiológica e vigor. Este é um dos testes de vigor recomendado pelo comitê de vigor da ISTA como padrão para a avaliação da qualidade de lotes de sementes de ervilha comum (HAMPTON \& TEKRONY, 1995).

Apesar de suas vantagens, o teste necessita de adequação da metodologia e estudos visando à utilização na rotina de avaliação de sementes de $\boldsymbol{P}$. sativum subsp. arvense, as quais diferem daquelas da ervilha comum quanto ao seu tegumento (espessura e cor), demonstrando dessa forma a necessidade do desenvolvimento de metodologia específica para essas sementes. Dentro desse contexto, este trabalho teve como objetivo adequar a metodologia do teste de condutividade elétrica para a avaliação da qualidade fisiológica de sementes de $\boldsymbol{P}$. sativum subsp. arvense.

\section{MATERIAL E MÉTODOS}

Este estudo foi realizado no Laboratório de Análise de Sementes do Departamento de Produção Vegetal da Faculdade de Ciências Agronômicas da Universidade Estadual Paulista "Júlio de Mesquita Filho" (FCA/UNESP), Campus de Botucatu, SP.

Dez lotes de sementes de $\boldsymbol{P}$. sativum subsp. arvense da cultivar 'APAR 83' foram avaliados, sendo identificados pelos números 5, 6, 7, 8, 9 10, 11, 12, 13 e 14. De acordo com as prescrições das Regras para Análise de Sementes (BRASIL, 2009), as amostras recém colhidas foram homogeneizadas. Após esse procedimento, as sementes foram acondicionadas em sacos de papel Kraft, em ambiente climatizado a $5^{\circ} \mathrm{C}$, durante três meses. Para fins de comparação e diferenciação da qualidade fisiológica dos lotes, antes do teste de condutividade elétrica, as sementes foram submetidas às seguintes avaliações: grau de umidade - realizada com duas subamostras de $5 \mathrm{~g}$ de sementes pelo método da estufa a $105^{\circ} \mathrm{C}$ por $24 \mathrm{~h}$ (BRASIL, 2009); massa de mil sementes - realizada com oito subamostras de 100 sementes por lote (BRASIL, 2009); teste de germinação - realizado com quatro subamostras de 50 sementes, conduzido em substrato rolo de papel, umedecidos com água destilada, na quantidade equivalente a 2,5 vezes o peso do substrato seco e incubados a $20^{\circ} \mathrm{C}$, no escuro, determinando-se a porcentagem de plântulas normais e anormais no quarto (primeira contagem) e sétimo dia após a semeadura (MACHADO, 2010); emergência de plântulas em campo (EPC) - conduzido em canteiros no interior de casa de vegetação telada, com parcelas constituídas 
por dois sulcos de $1,5 \mathrm{~m}$, espaçados de $0,2 \mathrm{~m}$, semeandose 50 sementes por sulco à profundidade de 4 a $5 \mathrm{~cm}$. As contagens foram realizadas diariamente até os 14 dias após a semeadura, quando foi calculada a porcentagem de emergência de plântulas em campo, conforme NAKAGAWA (1994); índice de velocidade de emergência (IVE) - calculado a partir dos dados de contagens diária de plântulas emergidas no teste de emergência e determinado pela da fórmula de MAGUIRE(1962).

Depois da caracterização da qualidade inicial dos lotes, efetuou-se o estudo da adequação da metodologia para condução do teste de condutividade elétrica, avaliando-se duas temperaturas para embebição $\left(20\right.$ e $\left.25^{\circ} \mathrm{C}\right)$, dois volumes de água (75 e $250 \mathrm{~mL}$ ) e cinco períodos de embebição: $8,16,20,24$ e 28 horas. Foram obtidas quatro amostras de 50 sementes, ao acaso, as quais foram pesadas com precisão de $0,001 \mathrm{~g}$ e em seguida foram colocadas em copos plásticos para embeber em água destilada nos volumes e períodos supracitados. Transcorridos os períodos programados para a embebição, determinouse a condutividade elétrica da solução com um condutivímetro de bancada. Após as leituras, calcularam-se os valores da condutividade elétrica por grama de sementes e os resultados expressos em $\mu \mathrm{S}$ $\mathrm{cm}^{-1} \mathrm{~g}^{-1}$ (VIEIRA \& KRZYZANOWSKI, 1999).

No procedimento estatístico, os dados obtidos nos testes de caracterização da qualidade inicial dos lotes de sementes $\boldsymbol{P}$. sativum subsp. arvense não foram transformados, sendo submetidos a análises de variância para todas as características avaliadas, as médias foram comparadas pelo teste de Scott-Knott a 5 e $1 \%$ de probabilidade. Para o teste de condutividade elétrica, foram conduzidos dois experimentos, sendo um com a embebição das sementes à temperatura de $20^{\circ} \mathrm{C}$ e o outro a $25^{\circ} \mathrm{C}$, utilizando o delineamento em blocos casualizados com parcelas subdivididas e quatro repetições. Nas parcelas, os tratamentos foram distribuídos no esquema fatorial 10x2, sendo 10 lotes e dois volumes de água, enquanto nas subparcelas avaliaram-se os períodos de embebição. Os dados não transformados foram submetidos à análise de variância a 5 e $1 \%$ de probabilidade pelo teste $F$, sendo as médias dos lotes comparadas pelo teste de Scott-Knott e dos volumes de água pelo teste de Tukey. Os dados referentes aos tempos de embebição, os quais também não foram transformados, foram submetidos à análise de regressão polinomial, testando-se as equações lineares e quadráticas, sendo aceitas as equações significativas até $5 \%$ de probabilidade pelo teste $\mathrm{F}$, com o maior coeficiente de determinação $\left(\mathrm{R}^{2}\right)$.

\section{RESULTADOS E DISCUSSÃO}

O grau de umidade inicial dos lotes de sementes de $\boldsymbol{P}$. sativum subsp. arvense variou de 14,0 a 14,6\% (Tabela 1). Embora na literatura não tenham

Tabela 1 - Médias dos dados de grau de umidade (U), massa de mil sementes, plântulas normais (PN), primeira contagem (PC), emergência de plântulas em campo (EPC), índice de velocidade de emergência (IVE) de dez lotes de sementes de ervilha forrageira. BotucatuSP, $2009^{1}$.

\begin{tabular}{|c|c|c|c|c|c|c|}
\hline Lotes & $\begin{array}{c}\mathrm{U} \\
(\%)\end{array}$ & $\begin{array}{l}\text { Massa de mil } \\
\text { sementes }(g)\end{array}$ & $\begin{array}{c}\text { Plântulas normais } \\
(\%)\end{array}$ & $\begin{array}{l}\mathrm{PC} \\
(\%)\end{array}$ & $\begin{array}{l}\text { EPC } \\
(\%)\end{array}$ & IVE \\
\hline 5 & 14,0 & $150,24 \mathrm{a}$ & $98 \mathrm{a}$ & $56 \mathrm{~b}$ & $86 \mathrm{~b}$ & $14,74 \mathrm{~b}$ \\
\hline 6 & 14,1 & $149,04 \mathrm{a}$ & $97 \mathrm{a}$ & $47 \mathrm{c}$ & $94 \mathrm{a}$ & $15,84 \mathrm{a}$ \\
\hline 7 & 14,1 & $107,44 \mathrm{f}$ & $95 \mathrm{~b}$ & $42 \mathrm{~d}$ & $88 \mathrm{~b}$ & $14,85 \mathrm{~b}$ \\
\hline 8 & 14,1 & $144,40 \mathrm{c}$ & $94 \mathrm{~b}$ & $66 \mathrm{a}$ & $87 \mathrm{~b}$ & $15,80 \mathrm{a}$ \\
\hline 9 & 14,2 & $136,66 \mathrm{~d}$ & $84 \mathrm{c}$ & $31 \mathrm{e}$ & $82 \mathrm{c}$ & $13,87 \mathrm{~b}$ \\
\hline 10 & 14,2 & $133,13 \mathrm{e}$ & $75 \mathrm{~d}$ & $38 \mathrm{~d}$ & $68 \mathrm{~d}$ & $11,06 \mathrm{~d}$ \\
\hline 11 & 14,6 & $96,03 \mathrm{~g}$ & $85 \mathrm{c}$ & $45 \mathrm{c}$ & $86 \mathrm{~b}$ & $14,94 \mathrm{~b}$ \\
\hline 12 & 14,0 & $94,05 \mathrm{~h}$ & $76 \mathrm{~d}$ & $44 \mathrm{c}$ & $80 \mathrm{c}$ & $13,17 \mathrm{c}$ \\
\hline 13 & 14,3 & $146,50 \mathrm{~b}$ & $100 \mathrm{a}$ & $42 \mathrm{~d}$ & $93 \mathrm{a}$ & $14,79 \mathrm{~b}$ \\
\hline 14 & 14,4 & $150,04 \mathrm{a}$ & $92 \mathrm{~b}$ & $32 \mathrm{e}$ & $89 \mathrm{~b}$ & $16,04 \mathrm{a}$ \\
\hline
\end{tabular}

${ }^{1}$ Médias seguidas pela mesma letra, na coluna, não diferem entre si pelo teste de Scott-Knott a 5\% de probabilidade.

Ciência Rural, v.41, n.6, jun, 2011. 
sido encontrados valores de teor de água de referência para sementes de $\boldsymbol{P}$. sativum subsp. arvense, os teores de água iniciais não foram ajustados, pois situaram-se dentro dos limites recomendados para a avaliação da condutividade elétrica de sementes de ervilha comum, que estaria entre 10 a 14\% (ISTA, 2006). O grau de umidade das sementes é um dos fatores que afetam os resultados do teste de condutividade elétrica e a padronização do teor de água das sementes é de fundamental importância, pois possibilita a obtenção de resultados uniformes (VIEIRA \& KRZYZANOWSKI, 1999).

Para os resultados da massa de 1000 sementes, verificou-se que as sementes dos lotes 5, 6 e 14 foram as que proporcionaram maior massa e as dos lotes 11 e 12 a menor massa (Tabela 1). Sementes com maiores tamanhos e densidades são as que possuem, normalmente, embriões bem formados e com maiores quantidades de reservas, potencialmente, as mais vigorosas (MARCOS FILHO, 2005).

Os dados referentes ao teste de germinação indicaram a maior porcentagem de germinação para as sementes dos lotes 5, 6 e 13, sendo que para aquelas dos demais lotes $(7,8,9,10,11,12$ e 14) os valores superaram $70 \%$, que é considerado o valor mínimo para a produção e comercialização de sementes de $\boldsymbol{P}$. sativum subsp. arvense para o Estado do Rio Grande do Sul (2000), sendo este o único Estado brasileiro que regulamentou normas para produção e comercialização de sementes dessa espécie. A avaliação do vigor, determinado pelo teste de primeira contagem da germinação (PC), ordenou os lotes em cinco níveis de vigor, identificando o lote 8 como o de melhor qualidade fisiológica e os lotes 9 e 14 como o de qualidade inferior (Tabela 1).

Pelos resultados do teste de emergência de plântulas em campo (EPC), constatou-se que os lotes 6 e 13 foram os de melhor qualidade fisiológica e o lote 10 como o de qualidade mais baixa (Tabela 1). Este fato deve-se aos diferentes níveis de qualidade fisiológica encontrados nos lotes de sementes de P. sativum subsp. arvense estudados e, como consequência, o comportamento das sementes em condições de campo foi distinto.
As avaliações do índice de velocidade de emergência (IVE) indicaram quatro níveis de vigor, classificando os lotes 6,8 e 14 com maior qualidade fisiológica e o lote 10 com qualidade fisiológica inferior (Tabela 1). Os testes de vigor, assim como o teste de germinação, foram sensíveis ao avaliar a qualidade das sementes dos diferentes lotes estudados, porém houve variações na ordenação dos lotes quanto ao vigor. Dessa forma é importante fazer uso de mais de um teste para determinar o vigor de lotes de sementes (MARCOS FILHO, 2005).

A metodologia recomendada pela Internacional Seed Testing Association (ISTA) para sementes de ervilha comum são amostras de 50 sementes, $250 \mathrm{~mL}$ de água deionizada a $20^{\circ} \mathrm{C}$ e leitura após 24 horas de embebição (ISTA, 2006). No entanto, VIEIRA \& KRZYZANOWSKI (1999) recomendaram amostras de 50 sementes, $75 \mathrm{~mL}$ de água deionizada a $25^{\circ} \mathrm{C}$ e leitura após 24 horas de embebição, de modo independente da espécie a ser avaliada. A recomendação da temperatura de $25^{\circ} \mathrm{C}$ para o acondicionamento caracteriza maior similaridade às condições climáticas brasileiras do que $20^{\circ} \mathrm{C}$, como recomendada pela ISTA(2006).

As médias e os gráficos dos dados obtidos para condutividade elétrica (CE) encontram-se, respectivamente, nas tabelas 2 e 3 e figura 1. Para ambas as temperaturas estudadas, em função do volume de água (Tabelas 2 e 3 e Figura 1), houve diluição da solução com o aumento do volume de água. Assim, com o uso do volume de $75 \mathrm{~mL}$, foram obtidos valores mais altos de condutividade elétrica em relação ao volume de $250 \mathrm{~mL}$, uma vez que a embebição em maior volume de água implica uma maior diluição dos lixiviados.

Dependendo da espécie, número de sementes, tempo de embebição e interações, pode-se recomendar distintos volumes de água para o teste de condutividade elétrica, com indicação de $75 \mathrm{~mL}$ para sementes de Arachis hypogaea L. (VANZOLINI \& NAKAGAWA, 2005) e $25 \mathrm{~mL}$ para sementes de Brassica oleracea L. (FESSEL et al., 2005).

Outra influência observada da quantidade de água sobre a condutividade elétrica das sementes foi a classificação dos lotes em níveis de vigor, 
Tabela 2 - Médias dos dados de condutividade elétrica de sementes de dez lotes de Pisum sativum subsp. arvense submetidas à embebição nas temperaturas de 20 e $25^{\circ} \mathrm{C}$, combinados a dois volumes de água ( 75 e $250 \mathrm{~mL}$ ), nos períodos de embebição (PE) de 8,16 e 20 horas. Botucatu-SP, $2009^{1}$.

\begin{tabular}{|c|c|c|c|c|c|c|c|c|c|c|c|c|c|c|c|}
\hline \multicolumn{8}{|c|}{ Temperatura de embebição $\left(20^{\circ} \mathrm{C}\right)$} & \multicolumn{8}{|c|}{ Temperatura de embebição $\left(25^{\circ} \mathrm{C}\right)$} \\
\hline \multirow{6}{*}{ PE } & \multirow{3}{*}{$\begin{array}{c}\text { Lote } \\
5\end{array}$} & \multicolumn{6}{|c|}{-------Volume de água (mL)------- } & \multirow{2}{*}{$\mathrm{PE}$} & \multirow{2}{*}{ Lote } & \multicolumn{6}{|c|}{------Volume de água (mL)---- } \\
\hline & & \multicolumn{3}{|c|}{75} & \multicolumn{3}{|c|}{250} & & & \multicolumn{3}{|c|}{75} & \multicolumn{3}{|c|}{250} \\
\hline & & 22,67 & $\mathrm{a}$ & $\mathrm{B}$ & 6,15 & $\mathrm{a}$ & A & & 5 & 24,68 & $\mathrm{a}$ & $\mathrm{B}$ & 8,02 & $\mathrm{a}$ & A \\
\hline & 6 & 23,19 & $\mathrm{a}$ & $\mathrm{B}$ & 7,12 & $\mathrm{a}$ & A & & 6 & 31,46 & $\mathrm{~b}$ & $\mathrm{~B}$ & 10,24 & $\mathrm{a}$ & A \\
\hline & 7 & 48,52 & $\mathrm{e}$ & $\mathrm{B}$ & 11,32 & $\mathrm{~b}$ & A & & 7 & 53,73 & $\mathrm{e}$ & B & 13,05 & $\mathrm{a}$ & A \\
\hline & 8 & 30,24 & $\mathrm{~b}$ & B & 8,19 & $\mathrm{a}$ & A & & 8 & 29,70 & $\mathrm{~b}$ & B & 10,33 & $\mathrm{a}$ & A \\
\hline \multirow[t]{10}{*}{8 horas } & 9 & 43,74 & $\mathrm{~d}$ & B & 10,71 & $\mathrm{~b}$ & A & 8 horas & 9 & 52,94 & $\mathrm{e}$ & B & 16,10 & $\mathrm{~b}$ & A \\
\hline & 10 & 44,35 & $\mathrm{~d}$ & B & 14,82 & $\mathrm{~b}$ & A & & 10 & 54,75 & $\mathrm{e}$ & B & 21,83 & $\mathrm{c}$ & A \\
\hline & 11 & 37,50 & $\mathrm{c}$ & B & 11,37 & $\mathrm{~b}$ & A & & 11 & 47,11 & $\mathrm{~d}$ & B & 14,77 & $\mathrm{~b}$ & A \\
\hline & 12 & 50,82 & $\mathrm{e}$ & B & 10,54 & $\mathrm{~b}$ & A & & 12 & 49,59 & $\mathrm{~d}$ & B & 16,80 & $\mathrm{~b}$ & A \\
\hline & 13 & 26,45 & $\mathrm{a}$ & B & 7,91 & $\mathrm{a}$ & A & & 13 & 30,18 & $\mathrm{~b}$ & B & 11,26 & $\mathrm{a}$ & A \\
\hline & 14 & 31,26 & $\mathrm{~b}$ & B & 7,84 & $\mathrm{a}$ & A & & 14 & 38,35 & $\mathrm{c}$ & B & 10,86 & $\mathrm{a}$ & A \\
\hline & 5 & 32,25 & $\mathrm{a}$ & B & 9,48 & $\mathrm{a}$ & A & & 5 & 35,18 & $\mathrm{a}$ & B & 11,97 & $\mathrm{a}$ & A \\
\hline & 6 & 37,62 & $\mathrm{~b}$ & B & 11,23 & $\mathrm{a}$ & A & & 6 & 40,81 & $\mathrm{~b}$ & B & 15,49 & $\mathrm{a}$ & A \\
\hline & 7 & 74,52 & $\mathrm{~h}$ & B & 17,24 & $\mathrm{~b}$ & A & & 7 & 69,35 & $\mathrm{e}$ & B & 18,78 & $\mathrm{~b}$ & A \\
\hline & 8 & 46,02 & $\mathrm{c}$ & B & 12,78 & $\mathrm{a}$ & A & & 8 & 42,76 & $\mathrm{~b}$ & B & 15,59 & $\mathrm{a}$ & A \\
\hline \multirow[t]{10}{*}{16 horas } & 9 & 51,26 & $\mathrm{~d}$ & B & 13,84 & $\mathrm{~b}$ & A & 16 horas & 9 & 79,57 & $\mathrm{f}$ & B & 23,75 & $\mathrm{c}$ & A \\
\hline & 10 & 64,17 & $\mathrm{f}$ & B & 25,41 & $\mathrm{c}$ & A & & 10 & 77,04 & $\mathrm{f}$ & B & 31,56 & $\mathrm{~d}$ & A \\
\hline & 11 & 56,80 & $\mathrm{e}$ & B & 15,81 & $\mathrm{~b}$ & A & & 11 & 64,60 & $\mathrm{~d}$ & B & 21,64 & $\mathrm{c}$ & A \\
\hline & 12 & 69,08 & $\mathrm{~g}$ & B & 15,93 & $\mathrm{~b}$ & A & & 12 & 65,57 & $\mathrm{~d}$ & B & 24,31 & $\mathrm{c}$ & A \\
\hline & 13 & 33,68 & $\mathrm{a}$ & B & 10,34 & $\mathrm{a}$ & A & & 13 & 37,65 & $\mathrm{a}$ & B & 15,28 & $\mathrm{a}$ & A \\
\hline & 14 & 41,15 & $\mathrm{~b}$ & B & 11,47 & $\mathrm{a}$ & A & & 14 & 52,60 & $\mathrm{c}$ & B & 14,74 & $\mathrm{a}$ & A \\
\hline & 5 & 35,26 & $\mathrm{a}$ & B & 10,59 & $\mathrm{a}$ & A & & 5 & 39,72 & $\mathrm{a}$ & B & 12,97 & $\mathrm{a}$ & A \\
\hline & 6 & 38,01 & $\mathrm{a}$ & B & 12,80 & $\mathrm{a}$ & A & & 6 & 44,49 & $\mathrm{~b}$ & B & 16,97 & $\mathrm{~b}$ & A \\
\hline & 7 & 80,46 & $\mathrm{f}$ & B & 19,50 & $\mathrm{~b}$ & A & & 7 & 76,01 & $\mathrm{f}$ & B & 20,02 & $\mathrm{~b}$ & A \\
\hline & 8 & 53,12 & $\mathrm{c}$ & B & 14,10 & $\mathrm{a}$ & A & & 8 & 49,77 & $\mathrm{c}$ & B & 17,10 & $\mathrm{~b}$ & A \\
\hline \multirow[t]{6}{*}{20 horas } & 9 & 60,22 & $\mathrm{~d}$ & B & 15,50 & $\mathrm{~b}$ & A & 20 horas & 9 & 87,40 & $\mathrm{~h}$ & B & 26,08 & $\mathrm{c}$ & A \\
\hline & 10 & 76,00 & $\mathrm{e}$ & B & 27,68 & $\mathrm{c}$ & A & & 10 & 83,20 & $\mathrm{~g}$ & B & 34,34 & $\mathrm{~d}$ & A \\
\hline & 11 & 62,93 & $\mathrm{~d}$ & B & 16,00 & $\mathrm{~b}$ & A & & 11 & 71,41 & $\mathrm{e}$ & B & 23,81 & $\mathrm{c}$ & A \\
\hline & 12 & 76,69 & $\mathrm{e}$ & B & 17,33 & $\mathrm{~b}$ & A & & 12 & 72,45 & $\mathrm{e}$ & B & 26,57 & $\mathrm{c}$ & A \\
\hline & 13 & 38,93 & $\mathrm{a}$ & B & 11,12 & $\mathrm{a}$ & A & & 13 & 40,81 & $\mathrm{a}$ & B & 16,71 & $\mathrm{~b}$ & A \\
\hline & 14 & 44,92 & $\mathrm{~b}$ & B & 12,28 & $\mathrm{a}$ & A & & 14 & 58,09 & $\mathrm{~d}$ & B & 16,04 & $\mathrm{~b}$ & A \\
\hline
\end{tabular}

${ }^{1}$ Médias seguidas pela mesma letra, dentro de cada tempo, maiúscula na linha, não diferem significativamente pelo teste de Tukey a $5 \%$ de probabilidade e minúscula na coluna não diferem significativamente pelo teste de Scott-Knott a $5 \%$ de probabilidade.

podendo-se notar que, para ambas as temperaturas de embebição, com o uso do volume de $75 \mathrm{~mL}$ de água, os lotes foram classificados em mais níveis de vigor, ou seja, este volume foi mais sensível para detectar diferenças de vigor do que o volume de $250 \mathrm{~mL}$ (Tabelas 2 e 3), devido à menor diluição.

Apesar de o volume de $75 \mathrm{~mL}$ classificar em mais níveis de vigor, deve-se sempre lembrar que, para ser avaliado como eficiência, um teste de vigor tem que proporcionar uma classificação dos lotes em diferentes níveis de vigor, de maneira proporcional à da emergência das plântulas no campo (MARCOS FILHO, 2005). Assim, o volume de $250 \mathrm{~mL}$ classificou os lotes aproximando-se da classificação da emergência em campo.

Nas duas temperaturas estudadas, observou-se aumento na quantidade de eletrólitos liberados pelas sementes de todos os lotes com o decorrer da embebição até o período de $28 \mathrm{~h}$; embora para alguns lotes e, principalmente, para o volume de $75 \mathrm{~mL}$ de água, os dados ajustaram-se a equações quadráticas (Figura 1). O aumento nos valores de

Ciência Rural, v.41, n.6, jun, 2011. 
Tabela 3 - Médias dos dados de condutividade elétrica de sementes de dez lotes de Pisum sativum subsp. arvense submetidas à embebição as temperaturas de 20 e $25^{\circ} \mathrm{C}$, combinados a dois volumes de água ( 75 e $\left.250 \mathrm{~mL}\right)$, nos períodos de embebição (PE) de 24 e 28 horas. Botucatu-SP, $2009^{1}$.

\begin{tabular}{|c|c|c|c|c|c|c|c|c|c|c|c|c|c|c|c|}
\hline \multicolumn{8}{|c|}{ Temperatura de embebição $\left(20^{\circ} \mathrm{C}\right)$} & \multicolumn{8}{|c|}{ Temperatura de embebição $\left(25^{\circ} \mathrm{C}\right)$} \\
\hline \multirow{2}{*}{$\mathrm{PE}$} & \multirow{3}{*}{$\begin{array}{l}\text { Lote } \\
5\end{array}$} & \multicolumn{6}{|c|}{----------'Volume de água $(\mathrm{mL})----------$} & \multirow{2}{*}{$\mathrm{PE}$} & \multirow{2}{*}{ Lote } & \multicolumn{6}{|c|}{-------Volume de água $(\mathrm{mL})$-- } \\
\hline & & \multicolumn{3}{|c|}{75} & \multicolumn{3}{|c|}{250} & & & \multicolumn{3}{|c|}{75} & \multicolumn{3}{|c|}{250} \\
\hline \multirow{8}{*}{24 horas } & & 38,43 & $\mathrm{a}$ & $\mathrm{B}$ & 11,00 & $\mathrm{a}$ & A & \multirow{8}{*}{24 horas } & 5 & 41,73 & $\mathrm{a}$ & $\mathrm{B}$ & 12,94 & $\mathrm{a}$ & A \\
\hline & 6 & 38,64 & $\mathrm{a}$ & $\mathrm{B}$ & 13,49 & $\mathrm{a}$ & A & & 6 & 47,16 & $\mathrm{~b}$ & $\mathrm{~B}$ & 18,18 & $\mathrm{~b}$ & A \\
\hline & 7 & 90,03 & $\mathrm{~g}$ & $\mathrm{~B}$ & 20,72 & $\mathrm{~b}$ & A & & 7 & 80,92 & $\mathrm{f}$ & $\mathrm{B}$ & 21,24 & $\mathrm{c}$ & A \\
\hline & 8 & 53,71 & $\mathrm{c}$ & B & 14,48 & $\mathrm{a}$ & $\mathrm{A}$ & & 8 & 52,49 & $\mathrm{c}$ & B & 18,15 & $\mathrm{~b}$ & A \\
\hline & 9 & 60,31 & $\mathrm{~d}$ & B & 16,36 & $\mathrm{~b}$ & A & & 9 & 94,33 & $\mathrm{~g}$ & $\mathrm{~B}$ & 28,02 & $\mathrm{~d}$ & A \\
\hline & 10 & 105,03 & $\mathrm{~h}$ & B & 31,78 & $\mathrm{c}$ & $\mathrm{A}$ & & 10 & 92,27 & $\mathrm{~g}$ & B & 38,71 & $\mathrm{e}$ & A \\
\hline & 11 & 65,45 & $\mathrm{e}$ & B & 17,02 & $\mathrm{~b}$ & A & & 11 & 72,94 & $\mathrm{e}$ & B & 23,87 & $\mathrm{c}$ & A \\
\hline & 12 & 84,70 & $\mathrm{f}$ & B & 18,09 & $\mathrm{~b}$ & A & & 12 & 74,95 & $\mathrm{e}$ & B & 28,41 & $\mathrm{~d}$ & A \\
\hline \multirow{12}{*}{28 horas } & 13 & 41,09 & $\mathrm{a}$ & B & 11,38 & $\mathrm{a}$ & $\mathrm{A}$ & \multirow{12}{*}{28 horas } & 13 & 46,16 & $\mathrm{~b}$ & $\mathrm{~B}$ & 18,67 & $\mathrm{~b}$ & A \\
\hline & 14 & 45,96 & $b$ & B & 13,29 & $\mathrm{a}$ & A & & 14 & 60,00 & $\mathrm{~d}$ & B & 16,79 & $\mathrm{~b}$ & A \\
\hline & 5 & 38,92 & $\mathrm{a}$ & B & 11,41 & $\mathrm{a}$ & A & & 5 & 42,07 & $\mathrm{a}$ & B & 13,41 & $\mathrm{a}$ & A \\
\hline & 6 & 41,61 & $\mathrm{a}$ & B & 15,54 & $\mathrm{~b}$ & A & & 6 & 47,89 & $\mathrm{~b}$ & B & 19,11 & $\mathrm{~b}$ & A \\
\hline & 7 & 93,35 & $\mathrm{f}$ & B & 21,33 & $\mathrm{c}$ & A & & 7 & 84,90 & $\mathrm{f}$ & B & 28,22 & $\mathrm{c}$ & A \\
\hline & 8 & 56,79 & $\mathrm{c}$ & B & 14,80 & $\mathrm{~b}$ & A & & 8 & 53,90 & $\mathrm{c}$ & B & 19,64 & $\mathrm{~b}$ & A \\
\hline & 9 & 82,84 & $\mathrm{~d}$ & B & 16,37 & $\mathrm{~b}$ & A & & 9 & 99,49 & $\mathrm{~g}$ & B & 29,76 & $\mathrm{~d}$ & A \\
\hline & 10 & 108,15 & $\mathrm{~g}$ & B & 31,82 & $\mathrm{~d}$ & $\mathrm{~A}$ & & 10 & 101,66 & $\mathrm{~g}$ & B & 42,94 & $\mathrm{e}$ & A \\
\hline & 11 & 80,65 & $\mathrm{~d}$ & B & 17,69 & $\mathrm{c}$ & A & & 11 & 77,53 & $\mathrm{e}$ & B & 25,80 & $\mathrm{c}$ & A \\
\hline & 12 & 87,09 & $\mathrm{e}$ & B & 19,59 & $\mathrm{c}$ & A & & 12 & 75,32 & $\mathrm{e}$ & B & 31,41 & $\mathrm{~d}$ & A \\
\hline & 13 & 42,19 & $\mathrm{a}$ & B & 11,73 & $\mathrm{a}$ & A & & 13 & 47,69 & $\mathrm{~b}$ & B & 20,80 & $\mathrm{~b}$ & A \\
\hline & 14 & 48,27 & $b$ & B & 13,72 & $\mathrm{~b}$ & A & & 14 & 62,56 & $\mathrm{~d}$ & B & 19,70 & $\mathrm{~b}$ & A \\
\hline
\end{tabular}

${ }^{1}$ Médias seguidas pela mesma letra, dentro de cada tempo, maiúscula na linha, não diferem significativamente pelo teste de Tukey a $5 \%$ de probabilidade e minúscula na coluna não diferem significativamente pelo teste de Scott-Knott a $5 \%$ de probabilidade.

condutividade elétrica com o tempo de embebição foi reportado por DIAS \& MARCOS FILHO (1996), trabalhando com sementes de Glycine max L., em que houve aumento nos valores de condutividade elétrica com o tempo de embebição, independentemente da qualidade do lote de sementes.

A maior lixiviação de exsudatos e condutividade elétrica foi constatada quando se utilizou a temperatura de $25^{\circ} \mathrm{C}$ e menor na de $20^{\circ} \mathrm{C}$ (Tabelas $2 \mathrm{e}$ 3 e Figura 1), provavelmente devido à diminuição da viscosidade da solução e maior mobilidade de íons na temperatura mais alta. Estes resultados divergem dos relatados por FESSEL et al. (2005) para sementes de Brassica oleracea L., uma vez que a lixiviação de exsudatos foi maior a 20 do que a $25^{\circ} \mathrm{C}$, embora deva-se levar em consideração que espécies diferentes podem responder de modo diverso aos tratamentos.

De acordo com os dados das tabelas 2 e 3 e figura 1, pode-se inferir que o volume de água, o tempo e a temperatura de embebição influenciaram os valores de condutividade elétrica, de forma que a adoção de subamostras de 50 sementes, $250 \mathrm{~mL}$ de água a $25^{\circ} \mathrm{C}$ por 24 horas possibilitam a melhor discriminação dos lotes em níveis de vigor.

Porém, deve-se ressaltar que a quantidade de água que melhor discrimina os lotes em níveis de vigor, apesar de ser recomendado pela ISTA para ervilha comum (ISTA, 2006), é considerada alta, uma vez que, considerando as repetições, a quantidade de água será de 1L por amostra, podendo-se tentar, para trabalhos futuros, estabelecer menores volumes, já que a economia de água é um fator fundamental.

\section{CONCLUSÃO}

O teste de condutividade elétrica utilizando $250 \mathrm{~mL}$ de água na temperatura de $25^{\circ} \mathrm{C}$ por 24 horas é promissor para a diferenciação de lotes de sementes de $\boldsymbol{P}$. sativum subsp. arvense.

Ciência Rural, v.41, n.6, jun, 2011. 


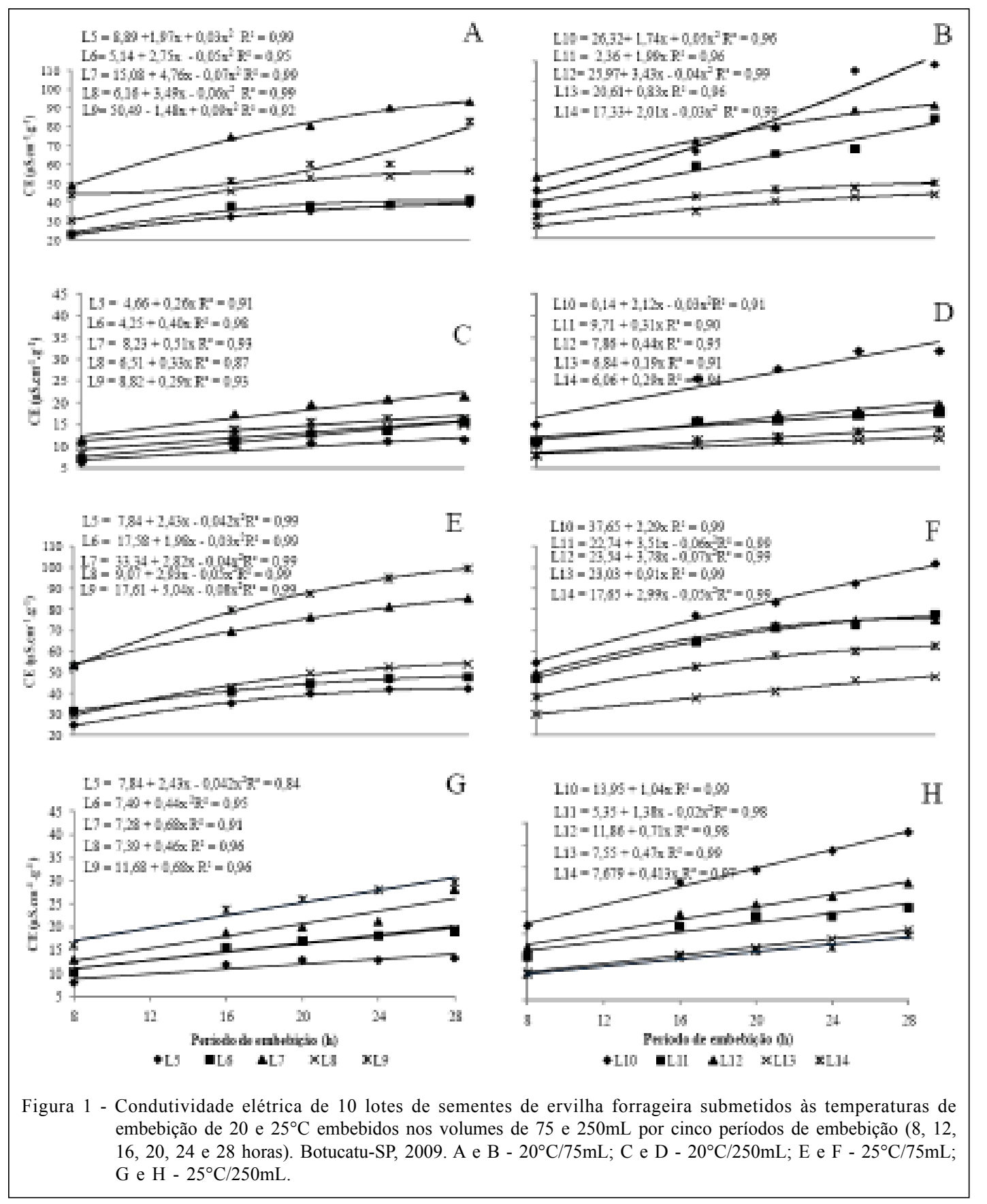

\section{REFERÊNCIAS}

BRASIL. Ministério da Agricultura, Pecuária e Abastecimento. Regras para análise de sementes. Brasília, 2009. 395p.

DIAS, D.C.F.S.; MARCOS FILHO, J. Testes de condutividade elétrica para avaliação do vigor de sementes de soja (Glycine max (L.) merrill). Scientia Agricola, Piracicaba, v.53, n.1, p.31-42, 1996. Disponível em: $<\mathrm{http}: /$ www.scielo.br/scielo.php?pid=S010390161996000100005\&script $=\mathrm{sci}$ arttext $>$. Acesso em: $17 \mathrm{jan}$. 2011. doi: 10.1590/S0103-90161996000100005.
FESSEL, S.A. et al. Teste de condutividade elétrica para avaliar a qualidade fisiológica de sementes de brócolis (Brassica oleracea L. var. italica Plenk). Científica, Jaboticabal, v.33, n.1, p.3541, 2005. Disponível em: <http://www.cientifica.org.br/ index.php/cientifica/article/view/63/44>. Acesso em: 15 out. 2010 .

GIORDANO L.B.; PEREIRA, A.S. A ervilha na alimentação animal. Informe Agropecuário, Belo Horizonte, v.14, n.158, p.65, 1989.

Ciência Rural, v.41, n.6, jun, 2011. 
HAMPTON, J.G.; TEKRONY, D.N. Controlled deterioration test. In: HAMPTON, J.G.; TEKRONY, D.N. Handbook of vigour test methods. Zürich: International Seed Testing Association, 1995. p.70-78.

INTERNATIONAL SEED TESTING ASSOCIATION International rules for seed testing. Zurich, 2006. 180p.

MACHADO, C.G. Métodos para a avaliação da qualidade fisiológica de sementes de ervilha forrageira (Pisum sativum subsp. arvense). 2010. 82f. Tese (Doutorado em Agronomia/Agricultura) - Universidade Estadual Paulista, Botucatu, SP.

MAGUIRE, J.D. Speed of germination-aid in selection and evaluation for seedling emergence and vigor. Crop Science, Madison, v.2, n.1, p.176-177, 1962.

MARCOS FILHO, J. Fisiologia de sementes de plantas cultivadas. Piracicaba: FEALQ, 2005. 495p.

NAKAGAWA, J. Testes de vigor baseados na avaliação das plântulas. In: VIEIRA, R.D.; CARVALHO, N.M. Testes de vigor em sementes. Jaboticabal: FUNEP, 1994. p.49-85.
ROSA, S.D.V.F. et al. Eficácia do teste de condutividade elétrica para uso em estudos de danos de secagem em sementes de milho. Revista Brasileira de Sementes, Brasília, v.22, n.1, p.5463, 2000. Disponível em: <http://www.abrates.org.br/revista/ artigos/2000/v22n1/artigo09.pdf >. Acesso em: 15 out. 2010.

RIO GRANDE DO SUL. Secretaria da Agricultura e Abastecimento. Departamento de Produção Vegetal. Normas e padrões de produção de sementes e mudas para o Estado do Rio Grande do Sul. Porto Alegre, 2000. 156p.

VANZOLINI, S.; NAKAGAWA, J. Teste de condutividade elétrica em sementes de amendoim. Revista Brasileira de Sementes, Pelotas, v.27, n.2, p.151-158, 2005. Disponível em: <http:// www.scielo.br/scielo.php?script=sci_arttext\&pid $=\mathrm{S} 0101$ $31222005000200022>$. Acesso em: 15 out. 2010. doi: 10.1590/ S0101-31222005000200022.

VIEIRA, R.D.; KRZYZANOWSKI, F.C. Teste de condutividade elétrica. In: KRZYZANOWSKI, F.C. et al. Vigor de sementes: conceitos e testes. Londrina: ABRATES, 1999. p.4.1-4.26.

VIEIRA, R.D.; CARVALHO, N.M. Testes de vigor em sementes. Jaboticabal: FUNEP, 1994. 164p. 